\title{
The spacer protein Spc110p targets calmodulin to the central plaque of the
}

\section{yeast spindle pole body}

\author{
Anne Spang, Katrin Grein and Elmar Schiebel* \\ Max-Planck Institut für Biochemie, Genzentrum, Am Klopferspitz 18A, 82152 Martinsried, Germany \\ *Author for correspondence
}

\section{SUMMARY}

Yeast calmodulin (CaM) was found to be localized to the microtubule organizing centre, the spindle pole body. The spindle pole body is a multi-layered structure consisting of outer, central and inner plaques. In this paper, we report that a fraction of $\mathrm{CaM}$ is in association with the central plaque of the spindle pole body. This localization is dependent on the calmodulin-binding site of another spindle pole body component, Spc110p, which serves as a spacer connecting the inner plaque with the central plaque. Since the CaM-binding site of Spc110p is located near the carboxy terminus, Spc110p-dependent localization of calmodulin defines the orientation of Spc110p with the carboxy terminus towards the central plaque and the amino terminus towards the inner plaque. This orientation of Spc110p was confirmed using antibodies specific for the amino-terminal end of Spc110p, which predominantly labelled the inner plaque. In addition, synthetic peptides corresponding to the calmodulin-binding site of Spc110p bound to calmodulin with a $K_{d}$ in the nanomolar range and nearly independent of $\mathrm{Ca}^{2+}$.

Key words: Calmodulin, Spc110p, Spindle pole body, Yeast

\section{INTRODUCTION}

In the yeast Saccharomyces cerevisiae, microtubule organizing functions are provided by the spindle pole body (SPB), a multilaminated organelle associated with the nuclear envelope throughout the life cycle of this organism (Byers and Goetsch, 1975). A number of SPB substructures can be discriminated by electron microscopy (Byers and Goetsch, 1975; Fig. 6). The inner and outer plaques of the SPB organize the cytoplasmic and nuclear microtubules, respectively. The central plaque is embedded in the nuclear envelope and is connected by fibres to the inner plaque of the SPB (Kilmartin et al., 1993; Rout and Kilmartin, 1990). The central plaque itself is divided by the central line into nuclear- and cytoplasmic-directed sections (Spang et al., 1993). Further, an intermediate line was observed in between the outer and central plaques of the SPB (Rout and Kilmartin, 1990). An additional SPB substructure is the half bridge, which appears as a darkly stained strip along the nuclear envelope on one side of the SPB (Byers and Goetsch, 1975). The half bridge has an important function in SPB duplication (Byers, 1981).

The fibres connecting the central with the inner plaque contain the essential Spc110p protein (Kilmartin et al., 1993). Spc110p was first described as a component of the yeast nucleoskeleton (Mirzayan et al., 1992). Further studies identified Spc110p as a component of the SPB (Kilmartin et al., 1993). Spc110p has a long central region with a predicted coiled-coil structure. Truncations in the coiled-coil domain of
Spc110p change the spacing between the central and inner plaques of the SPB (Kilmartin et al., 1993). Recently, a calmodulin $(\mathrm{CaM})$ binding site was identified within the carboxy-terminal domain of Spc110p. Mutations in the CaMbinding site of Spc110p, which abolish $\mathrm{CaM}$ binding in vitro, are lethal for cells. However, the total deletion of the CaMbinding domain of Spc110p does not affect viability (Geiser et al., 1993; Stirling et al., 1994). This result may be explained by an auto-inhibitory function of the $\mathrm{CaM}$ binding site, analogous to myosin light chain kinase (Yano et al., 1993) or $\mathrm{Ca}^{2+} / \mathrm{CaM}-d e p e n d e n t$ protein kinase (Schulman et al., 1992).

Some mutants in the structural gene of CaM (CMD1) like cmdl-1 and cmd1-101 show mitotic defects (Davis, 1992; Ohya and Botstein, 1994). In the case of $c m d l-1$, this defect is suppressed by deleting the CaM-binding site near the carboxy terminus of Spc110p (Geiser et al., 1993). This suggests that Spc110p is one of the essential targets of CaM in yeast. Remarkably, CaM fulfils its essential functions without $\mathrm{Ca}^{2+}$-binding (Geiser et al., 1991). Based on this finding it has been suggested that the interaction of $\mathrm{CaM}$ with essential targets is $\mathrm{Ca}^{2+}$-independent. Therefore the $\mathrm{Ca}^{2+}$-dependent binding of $\mathrm{CaM}$ to Spc110p in an in vitro assay was surprising (Spang et al., 1995). Furthermore, while the interaction of CaM with the SPB has been demonstrated (Geiser et al., 1993; Stirling et al., 1994), it is unknown whether this localization is dependent on Spc110p, which is expected when CaM binds to the SPB via Spc110p. In this case, a deletion of the CaM-binding site of Spc110p should severely affect the localization of CaM to the SPB. 
Here we report that $\mathrm{CaM}$ is a component of the central plaque of the SPB. This association is dependent on the CaMbinding site of Spc110p. The Spc110p-dependent localization of $\mathrm{CaM}$ with the central plaque defines the orientation of Spc110p in the SPB with the CaM-binding site at the carboxyterminal end of Spc110p near the central plaque. In agreement with this conclusion, an antibody specific for the amino terminus of Spc110p predominantly labelled the inner plaque of the SPB, confirming that Spc110p is an elongated protein connecting the central with inner plaques. In addition, by an in vitro assay we could demonstrate that $\mathrm{CaM}$ binds to the CaM-binding site of Spc110p with a $K_{d}$ in the nanomolar range.

\section{MATERIALS AND METHODS}

\section{Media, bacterial transformation, DNA techniques}

Media for growth of yeast were as described by Guthrie and Fink (1991). DNA manipulations were performed as described by Sambrook et al. (1989).

\section{Cloning of SPC110, SPC110 disruption cassette, GST- SPC110 gene fusion}

SPC110 was cloned by PCR using primers NUF1-11 (5'CGTGGGATCCGGAGCTCCTTGTAGACA-3') and NUF1-14 (5'CGTGGGATCCGAAGTTCAAAGAACTCC-3'; Mirzayan et al., 1992). Chromosomal DNA from strain YPH499 (Sikorski and Hieter, 1989) was used as a template. Primers NUF1-11 and NUF1-14 each carry a BamHI restriction site. The $3.48 \mathrm{~kb}$ PCR fragment was cloned into plasmid pBluescript SK (Stratagene; pSM104). The 3.48 BamHI fragment of pSM104 was cloned into the URA3- or TRP1-based vectors pRS316 or pRS314 (Sikorski and Hieter, 1989) to give plasmids pCM94 and pCM132, respectively. spc110-407 of plasmid pJG94 (Geiser et al., 1993) on a $3.3 \mathrm{~kb} P v u I I-S a l$ fragment was inserted into the SmaI-SalI restriction sites of pRS314 (pSM237).

For the construction of the SPC110 disruption cassette of pSM249, plasmid pSM104 was restricted with NsiI and SnaBI, which removes $76 \%$ of the coding region of SPC110 including the region that encodes the CaM-binding site. The HIS3 gene on a PstI-SmaI fragment was cloned into the NsiI-SnaBI restriction sites of pSM104.

The construction of GST-N-SPC110 (pSM72) and GST-C-SPC110 (pSM74) in vector pGEX-3X (Pharmacia) has been described (Spang et al., 1995). Plasmid pSM236 carrying GST-C-spc110-407 on pGEX$3 \mathrm{X}$ was constructed identically.

\section{Constructions of yeast strains}

Yeast strain YPH500 (Sikorski and Hieter, 1989) was transformed with plasmid pCM94 carrying SPC110 on plasmid pRS316 (ESM195). The chromosomal SPC110 of ESM195 was disrupted by the one step gene replacement method of Rothstein (1983) using the SPC110 disruption cassette of pSM249 (4spc110::HIS3).

Strain ESM211 was transformed with plasmids pSM237 (ESM2141) or pCM132 (ESM213-1). Strains ESM214-1 and ESM213-1 were incubated on 5-fluoroorotic acid (5-FOA) plates to select for colonies which spontaneously lost pCM94. SPC110 function is now provided by either SPC110 (ESM213-2) or SPC110-407 (ESM214-2) on plasmid pRS314.

\section{Affinity-purified CaM antibodies}

CMD1 was amplifed by PCR as described by Spang et al. (1995) and cloned into pRS315 (pCM137). CMD1 of pCM137 was amplified by PCR using primers CaM-BamHI (5'-CGTGGGATCCTCCAATCTTACCGA-3') and T7 (5'-AATACGACTCACCATAG-3'). Primer CaM-BamHI introduces a BamHI restriction site upstream of the translation initiation codon of $C M D 1$. The PCR product was restricted with $\mathrm{BamHI}$ and $\mathrm{Sna \textrm {BI }}$ and cloned into vector pGEX-4T-3 (Pharmacia, pSM178). Recombinant GST-CaM fusion protein was isolated from E. coli BL21 (DE3) (Tabor and Richardson, 1985) carrying pSM178, as described by Spang et al. (1993). GST-CaM was injected into rabbits as described by Harlow and Lane (1988). Affinity purified anti-CaM antibodies were obtained as described by Spang et al. (1993) using purified, recombinant $\mathrm{CaM}$.

\section{Nuclei isolation, SPB preparation, cell extracts}

Nuclei and SPBs from strains YPH499 and ESM214-2 were isolated as described by Rout and Kilmartin (1990). Protein extracts were prepared according to Ausubel et al. (1994).

\section{Immunoelectron microscopy, immunofluorescence of yeast cells}

Immunoelectron microscopy of whole yeast cells and isolated SPBs was performed with affinity-purified rabbit anti-N-Spc110p (Spang et al., 1995), anti-CaM or mouse monoclonal anti- $\beta$-tubulin antibodies (WA3), according to Preuss et al. (1991). Gold particles bound to protein A or rabbit anti-mouse IgGs (BioCell) were visualized in a Jeol JEM 100B electron microscope. No signal was obtained when cells were incubated either with secondary antibodies only or with preimmune serum.

Immunofluorescence of yeast cells was performed as described by Adams and Pringle (1989) or Rout and Kilmartin (1990). Affinitypurified anti-N-Spc110p and anti-CaM antibodies were used. Secondary antibodies were goat anti-rabbit IgG and rabbit anti-goat $\mathrm{IgG}$, both conjugated with $\mathrm{Cy} 3$ (Jackson ImmunoResearch Laboratories). No signal was obtained when cells were incubated either with secondary antibodies only or with preimmune serum.

\section{Immunoblotting, protein determination}

Protein concentration of samples was determined by the method of Bradford (1976). Proteins were separated by SDS-PAGE (Laemmli, 1970). Immunoblotting was performed as described by Spang et al. (1993). Detection was made by enhanced chemilumenscence. The primary antibodies were rabbit anti-CaM and goat anti-N-Spc110p (Spang et al., 1995). Secondary antibodies were goat anti-rabbit IgG (Bio-Rad), rabbit anti-goat $\mathrm{IgG}$ and donkey anti-goat (Jackson ImmunoResearch Laboratories) conjugated with horseradish peroxidase.

\section{Purification of CaM, binding of CaM to synthetic peptides, overlay assay}

Yeast CaM was expressed from the phage $\mathrm{T} 7$ promoter in E. coli BL21 (DE3, pSM71) (Studier and Moffat, 1986; Tabor and Richardson, 1985) as described by Spang et al. (1995), and purified according to $\mathrm{H}$. Wiech et al. (unpublished). Briefly, after a temperature precipitation step $\left(10\right.$ minutes, $\left.80^{\circ} \mathrm{C}\right)$ the supernatant was applied onto a DEAE-Cellulose column (Sigma). CaM-containing fractions were further enriched by a Phenyl Sepharose step (high performance; Pharmacia) and finally purified using a Superdex 75 HiLoad 16/60 column on a FPLC system (Pharmacia).

The synthetic peptides RRWSFKTVALLVLACVMKRI and RRLSFKTVAWLVLASVRMKRI were synthesized by the MaxPlanck Peptide Service. The molecular mass of the C8 reverse-phase HPLC-purified peptides was confirmed by mass spectrometry. The binding of the synthetic peptides to purified yeast $\mathrm{CaM}$ was determined by tryptophan fluorescence in $\mathrm{Ca}^{2+}$-buffers (Molecular Probes, Eugene, OR) containing $10 \mathrm{mM}$ MOPS, pH 7.2, $100 \mathrm{mM} \mathrm{KCl}$ and 1 $\mathrm{mM}$ DTT. The excitation wavelength was $295 \mathrm{~nm}$. The emission was measured at $330 \mathrm{~nm}$. The $K_{\mathrm{d}}$ values were calculated using the program Psi-Plot. $\left.{ }^{35} \mathrm{~S}\right] \mathrm{CaM}$ was labeled and purified as described by Spang et al. (1995). The overlay assay was performed in the presence of $1 \mathrm{mM}$ $\mathrm{Ca}^{2+}$ (Spang et al., 1995). 


\section{RESULTS}

\section{$\mathrm{CaM}$ is a component of the central plaque of the SPB}

CaM was localized to the SPB by indirect immunofluorescence using special fixation conditions (Geiser et al., 1993; Stirling et al., 1994) that preserve SPB antigens (Rout and Kilmartin, 1990). To understand the function of CaM at the SPB, it is important to determine its association with substructures of the SPB. Functions for some SPB substructures were deduced from ultrastructural analysis (Byers, 1981). Most interestingly, the inner and central plaques are connected by fibres that contain Spc110p, which carries a CaM-binding site at its carboxy-terminal end (Geiser et al., 1993; Kilmartin et al., 1993).

For these localization studies, we prepared affinity-purified anti-CaM antibodies. The specificity of the affinity-purified anti-CaM antibodies was tested by immunoblotting using a total yeast extract. The anti-CaM antibodies recognized only one protein of about $16 \mathrm{kDa}$, the predicted molecular mass of $\mathrm{CaM}$ (Fig. 1A, lane 1). This protein revealed the same migration behaviour as recombinant $\mathrm{CaM}$ (lane 4). Cdc31p, another $\mathrm{Ca}^{2+}$-binding protein, which is $38 \%$ identical to $\mathrm{CaM}$, was not detected by the antibodies (lane 3), confirming that our affinity-purified anti-CaM antibodies are specific for CaM. Using the anti-CaM antibodies, we observed the same cellular distribution of $\mathrm{CaM}$ in yeast cells as has been reported before (data not shown; Brockerhoff and Davis, 1992). CaM was mainly localized at the sites of bud growth, but also faintly distributed throughout the cell. In addition, SPB staining was observed using the fixation conditions described by Rout and Kilmartin (1990) (Fig. 2C).

Next, the localization of CaM was investigated by immunoelectron microscopy using isolated SPBs (Rout and Kilmartin, 1990). Enriched fractions of SPBs contain about 500-fold more SPBs per volume than yeast cells, making it much easier to obtain a representative view of the localization of a SPB component. More than 1,000 SPBs were investigated, of which about $45 \%$ were stained with gold particles. The labelling of only $45 \%$ of the SPBs is explained by the post-embedding labelling technique, where only epitopes exposed on the surface of the section are accessible for detection by the antibody. The outer and central plaques were clearly detectable in the isolated SPBs. The central plaque was disrupted by the central line

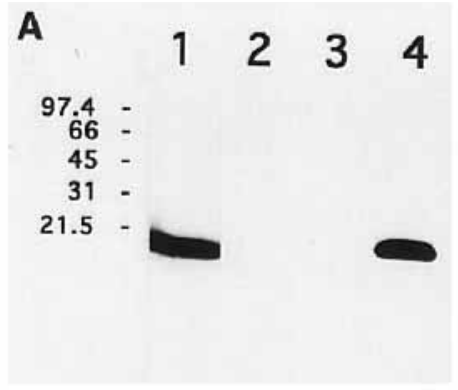

Fig. 1. CaM is a component of the central plaque of the SPB. (A). The anti-CaM antibodies are specific. Total yeast extract (80 $\mu \mathrm{g}$; lane 1$), E$. coli extracts ( $80 \mu \mathrm{g}$ each) expressing no recombinant protein (lane 2), Cdc31p (lane 3) or CaM (lane 4), were separated by SDS-PAGE, transferred onto a nitrocellulose membrane and incubated with affinity-purified anti-CaM antibodies. The molecular masses of marker proteins are indicated in $\mathrm{kDa}$. (B-F) Immunoelectron microscopy of isolated SPBs. Ultrathin sections of isolated SPB were incubated with affinitypurified rabbit anti-CaM antibodies followed by Protein A-gold. The gold particles have a diameter of $10 \mathrm{~nm}$. The small arrowheads in (B,D and E) indicate the position of gold particles associated with microtubules. Large arrowheads: c, central plaque; cl, central line; i, inner plaque; il, intermediate line; $\mathrm{m}$, microtubules; o, outer plaque. Bars: (B,C,D) 50 $\mathrm{nm}$; (E,F) $125 \mathrm{~nm}$.

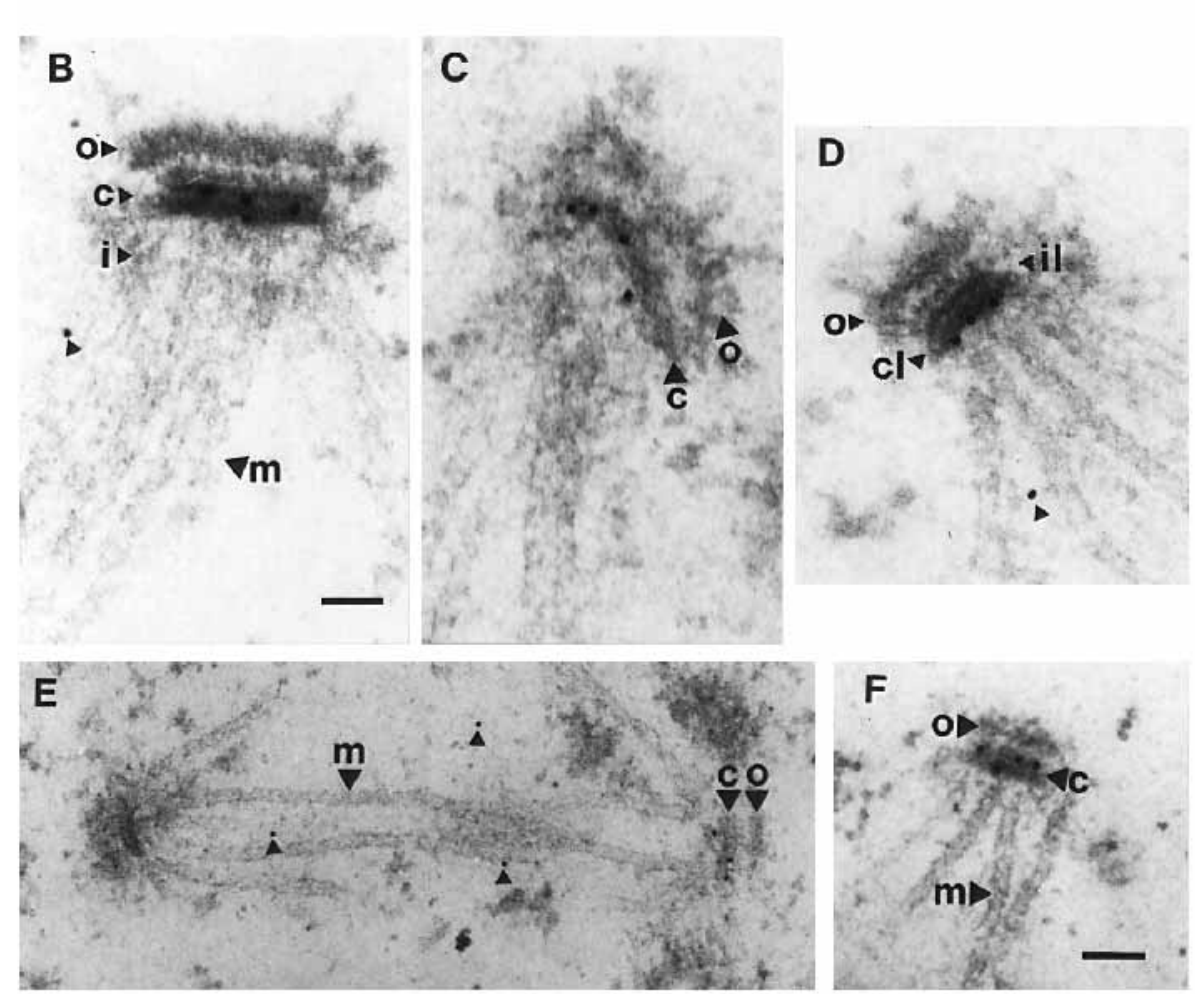


Fig. 2. The localization of $\mathrm{CaM}$ to the central plaque is dependent on the CaM-binding site of Spc110p. (A) CaM does not interact with Spc110-407p. GST-CSpc110-407p (lanes 1 and 4), GST-C-Spc110p (lanes 2 and 5) and GST-N-Spc110p (lanes 3 and 6) were expressed in E. coli and separated by SDS-PAGE. The expression of the recombinant proteins was tested by Coomassie Blue staining (lanes 1 to 3 ). The recombinant proteins are indicated by the asterisk. Lanes 4 to 6 were transferred onto a nitrocellulose membrane. The membrane was incubated with $\left[{ }^{35} \mathrm{~S}\right] \mathrm{CaM}$ and subsequently subjected to autoradiography. The molecular masses of marker proteins are indicated in $\mathrm{kDa}$. (B) spc110-407 is expressed in yeast. An immunoblot of yeast extracts $(80 \mu \mathrm{g})$ from wild-type cells (lane 1) and cells expressing spc110-407 (ESM2142 ; lane 2) were incubated with affinity-purified goat anti$\mathrm{N}$-Spc110p antibodies. (C) CaM is not associated with SPBs of spc110-407 cells. SPC110 and spc110-407 cells were fixed with acetone and methanol (Rout and Kilmartin, 1990) and incubated with mouse monoclonal anti-90-kDa and rabbit anti-CaM antibodies. Secondary antibodies were goat anti-mouse IgGs coupled to $\mathrm{Cy} 3$ or goat anti-rabbit IgGs coupled to FITC. Cells were analyzed by a Zeiss Axiovert 134 confocal microscope. The green signal indicates the localization of CaM. The red signal corresponds to the localization of the $90 \mathrm{kDa}$ SPB protein. The yellow signal in SPC110 cells results from the colocalization of $\mathrm{CaM}$ and the $90 \mathrm{kDa} \mathrm{SPB}$ protein. (D) $\mathrm{CaM}$ is not associated with $\mathrm{SPBs}$ from spc110-407 cells. Immunoelectron microscopy of isolated SPBs from spc110-407 cells. A double-labelling experiment was performed with affinity-purified rabbit anti-CaM (15 nm gold) and mouse monoclonal anti- $\beta$ tubulin antibodies ( $5 \mathrm{~nm}$ gold). The arrowheads point to the $5 \mathrm{~nm}$ gold particles. Note, no $15 \mathrm{~nm}$ gold particles $(\mathrm{CaM})$ associated with SPBs from spc110-407 cells were observed, indicating that the localization of $\mathrm{CaM}$ was dependent on the CaM-binding site of Spc110p. c, central plaque; i, inner plaque; o, outer plaque. Bars: (C) $5 \mu \mathrm{m}$; (D) $50 \mathrm{~nm}$.
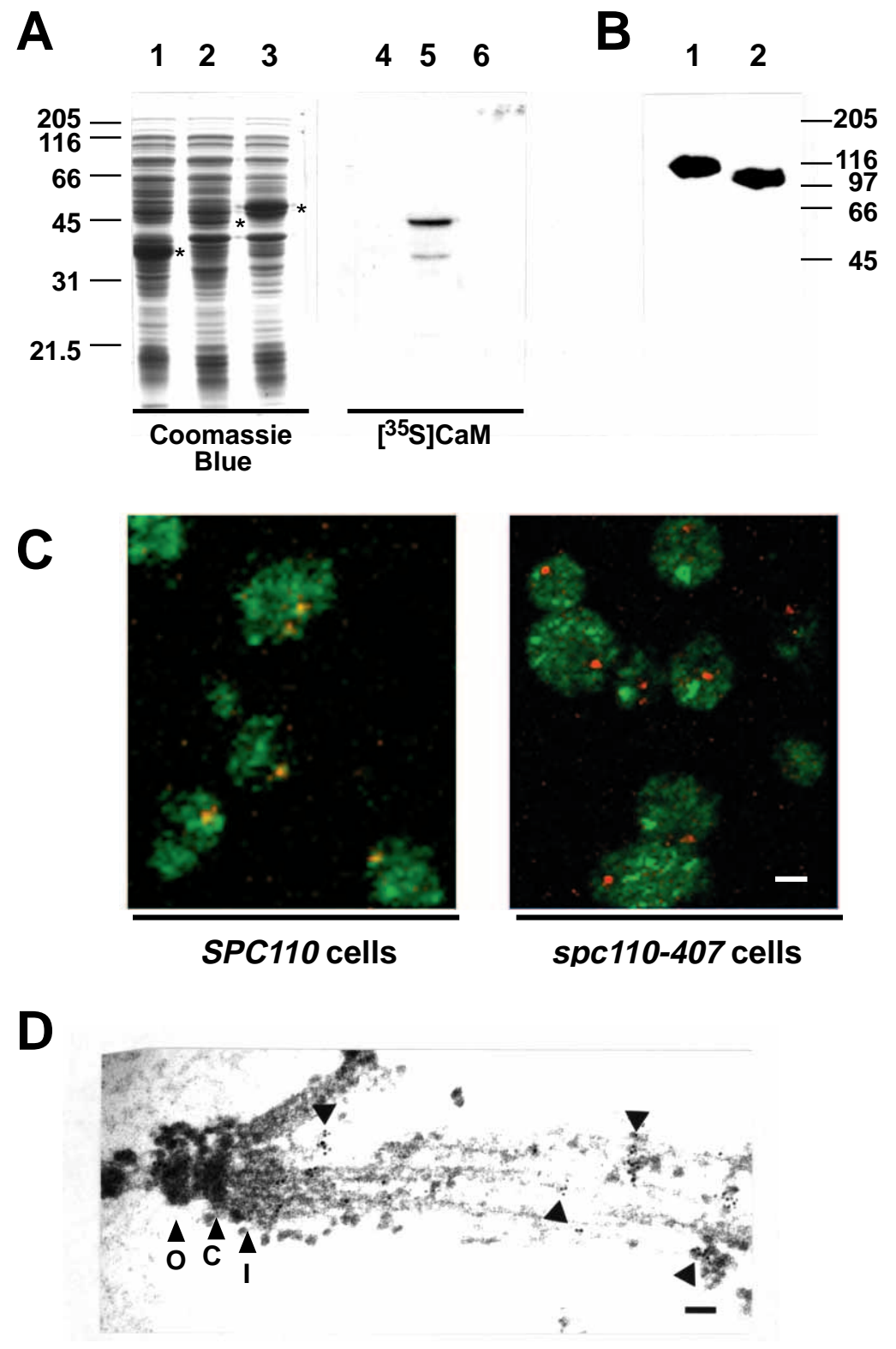

\section{Interaction of CaM with Spc110p is required for its localization to the SPB}

Spc110p possesses a CaM-binding site at its carboxy-terminal domain (Geiser et al., 1993; Stirling et al., 1994). However, whether CaM binds to the SPB via Spc110p or an additional SPB component has not been investigated. To test this, we used spc110-407, which contains a frame-shift mutation that converts codon 864 to a stop codon, thereby deleting the CaMbinding site of Spc110p (amino acids 900 to 916; Geiser et al., 1993; Stirling et al., 1994). We first confirmed that CaM does not interact with Spc110-407p. Therefore, GST-C-Spc110407p (Fig. 2A, lane 1; fusion protein is marked by an asterisk), GST-C-Spc110p (lane 2) and GST-N-Spc110p (lane 3) were produced in E. coli. While CaM bound to the carboxy-terminal domain of Spc110p (GST-C-Spc110p; lane 5), no interaction was observed with GST-C-Spc110-407p (lane 4) or the aminoterminal portion of Spc110p fused to GST (GST-N-Spc110p; 
A
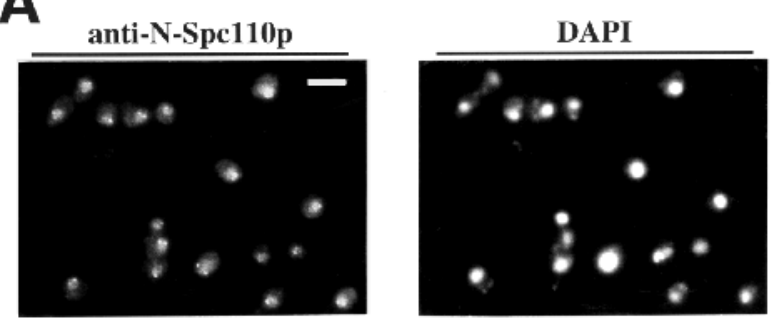

B

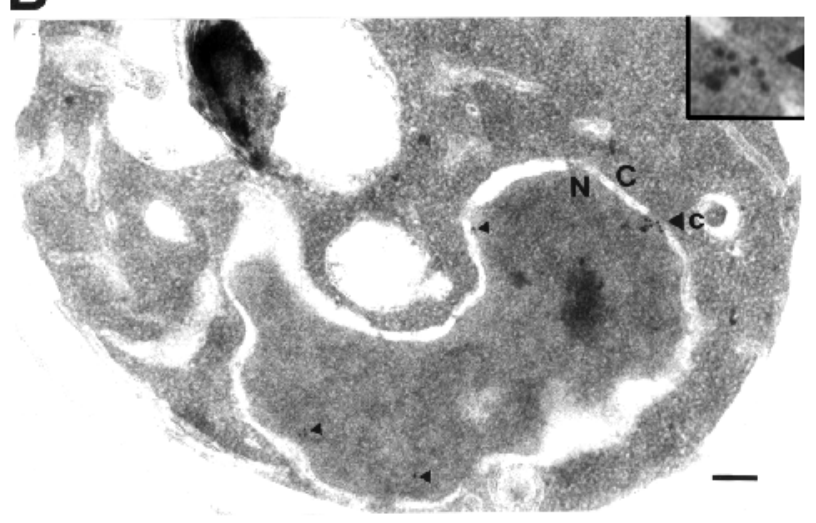

C

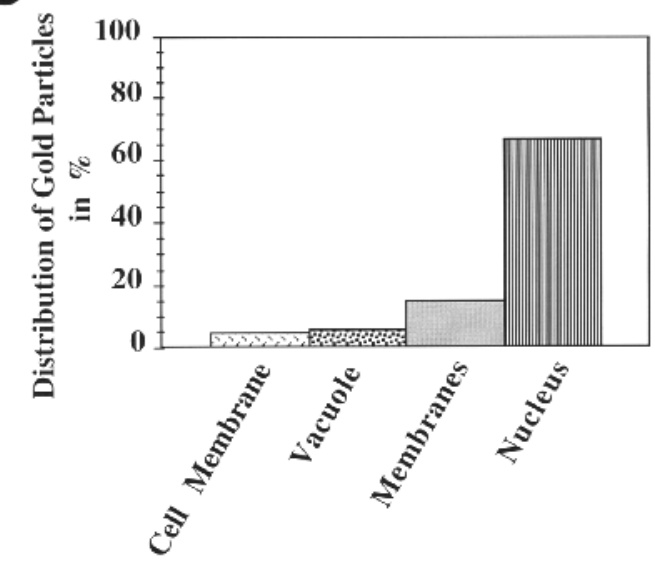

Fig. 3. Spc110p signal is also present in the nucleus.

(A) Immunofluorescence of yeast cells with affinity-purified rabbit anti-N-Spc110p antibodies. DNA was stained with DAPI.

(B) Immunoelectron microscopy of yeast cells with affinity-purified rabbit anti-N-Spc110p antibodies. The labelling was visualized with Protein A-gold (10 nm). The small arrowheads point to the gold particles distributed throughout the nucleus. The large arrowhead indicates the position of the central plaque of the SPB. Inset in B is a threefold enlargement of the SPB with Spc110p signal on the nuclear side of the central plaque. c, central plaque; C, cytoplasm; N, nucleus. (C) The distribution of the Spc110p signal in sections of 100 cells was determined. 'Membranes' indicates the gold particles associated with membranous structures of uncertain origin and 'Cell Membranes' the gold particles associated with the cytoplasmic membrane. Bars: (A) $5 \mu \mathrm{m}$; (B) $0.36 \mu \mathrm{m}$.

lane 6). We then constructed strain ESM214-2 carrying the spc110-407 allele. This was possible, since the deletion of the CaM-binding site of Spc110p does not affect viability (Stirling et al., 1994). We confirmed the truncation of Spc110p in spc110-407 cells. In agreement with a deletion of the 86 carboxy-terminal amino acids of Spc110p, the electrophoretic mobility of Spc110-407p (Fig. 2B; lane 2) was increased in comparison to Spc110p (lane 1).

SPC110 and spc110-407 cells were fixed with acetone and methanol and incubated with antibodies against the $90 \mathrm{kDa}$ SPB component (red colour in Fig. 2C) and CaM (green colour). In these experiments cytoplasmic CaM was not clearly localized with the sites of bud growth (Brockerhoff and Davis, 1992), explained by the acetone-methanol fixation, which does not preserve all substructures of a yeast cell. A fraction of CaM was associated with the SPB in SPC110 cells, indicated by the yellow colour resulting from an overlap of the red anti-90-kDa and green anti-CaM signals. In contrast, the SPB of spc110-407 cells was only stained by the anti-90$\mathrm{kDa}$ antibodies (red), suggesting that $\mathrm{CaM}$ was not associated with these SPBs.

The same result was obtained using enriched SPBs from spc110-407 cells. While in all inspected SPBs $(n=1,000)$ the attached microtubules were labelled by the anti-tubulin antibody ( $5 \mathrm{~nm}$ gold particles; arrowheads), no CaM signal (15 $\mathrm{nm}$ gold) was detected at the SPB (Fig. 2D). Taken together, our results suggest that CaM binds to the SPB via the carboxyterminal CaM-binding site of Spc110p.

\section{The amino-terminal domain of Spc110p is close to the inner plaque of the SPB}

The Spc110p-dependent association of CaM with the central plaque suggests that the CaM-binding site, and thereby the carboxy-terminal domain of Spc110p, is in close proximity with the central plaque of the SPB. Since it has been suggested that Spc110p is a filamentous protein connecting the central plaque with the inner plaque (Kilmartin et al., 1993), the amino-terminal domain of Spc110p may associate with the inner plaque. We tested this hypothesis by immunoelectron microscopy using affinity-purified anti-Spc110p antibodies, which were directed against the amino-terminal 175 amino acids of Spc110p (Spang et al., 1993).

As reported before (Rout and Kilmartin, 1990), our anti-NSpc110p antibody detected Spc110p at the SPB of yeast cells by indirect immunofluorescence (Fig. 3A). Additionally, Spc110p signal was observed throughout the nucleus (Fig. 3A, compare anti-N-Spc110p with DAPI). Interestingly, depending on the manner of fixation of yeast cells, Spc110p has been described as component of the SPB (Rout and Kilmartin, 1990), the nucleoskeleton (Mirzayan et al., 1992), or the SPB and the nucleus (Kilmartin et al., 1993).

The dual localization of Spc110p was confirmed by immunoelectron microscopy of cells using the anti-N-Spc110p antibodies. The SPB appeared by the fixation and embedding technique of Preuss et al. (1991) as a discontinuity of the nuclear envelope by the central plaque (Fig. 3B; large arrowhead points to the central plaque; Spang et al., 1993). The central line interrupting the central plaque was clearly detectable. Outer and inner plaques as well as microtubules were not resolved by this technique (Spang et al., 1993). SPBs were labelled by the anti-N-Spc110p antibodies. Up to six gold particles were located on the nuclear side of the central plaque. Besides SPB staining, gold particles were distributed over the entire cell (Fig. 3B). We quantified the localization of Spc110p-signal in 100 sections of cells (Fig. 3C), not counting 
Fig. 4. The amino terminus of Spc110p is directed towards the inner plaque of the SPB. (A-D) Immunoelectron microscopy of isolated SPB. Ultrathin sections of isolated SPBs were incubated with affinity-purified rabbit anti-N-Spc110p antibodies followed by Protein A-gold (10 nm). (E-F) Spc110-407p is associated with the SPB. Immunoelectron microscopy of SPBs from spc110-407 cells using anti-N-Spc110p antibodies. c, central plaque; i, inner plaque; il, intermediate line; $\mathrm{m}$, microtubules; o, outer plaque. Bars: (A-D) $50 \mathrm{~nm}$; (E) $50 \mathrm{~nm}$.
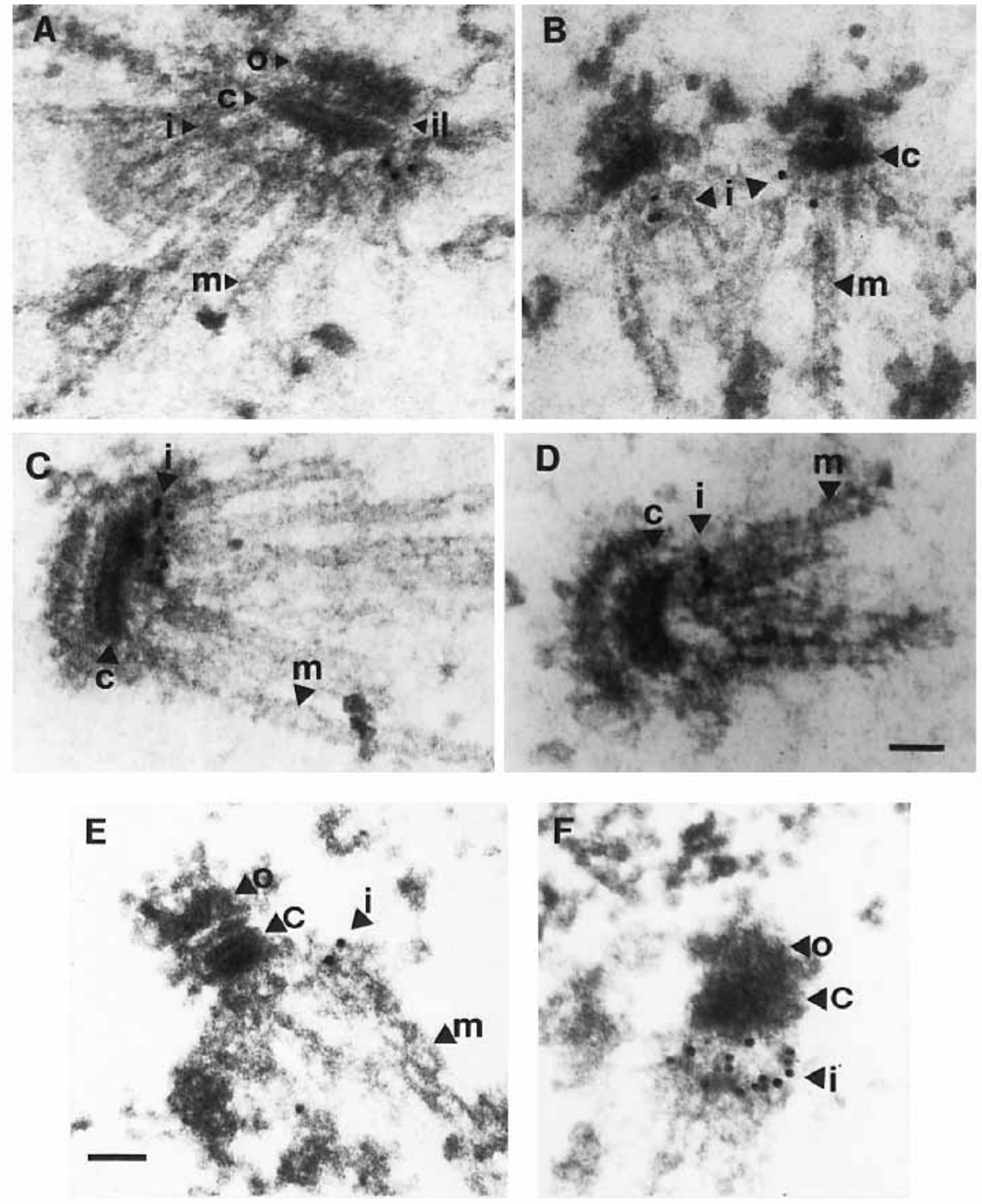

the SPB signals. About $75 \%$ of the Spc110p signal was found in the nucleus. The remaining signal was associated with either the vacuole $(3 \%)$, the cytoplasmic membrane (2\%; 'Cell Membranes'; Fig. 3C) or membranous structures of uncertain origin (20\%; 'Membranes').

Next, ultrathin sections of enriched SPB were subjected to immunoelectron microscopy with anti-N-Spc110p antibodies. About 1,000 SPBs were inspected, of which $40 \%$ were associated with gold particles. In about $85 \%$ of the SPBs with a Spc110p signal, 2-10 gold particles were found close to or directly at the inner plaque (Fig. 4A to D). In most of the other cases $(10 \%)$, the signal was located between the central and inner plaques but never at the central plaque. The remaining gold particles $(5 \%)$ were associated with other SPB substructures. No signal was observed when the first antibody was omitted, showing that the labelling was dependent on the antiN-Spc110p antibody.

We tested whether the deletion of the CaM-binding site of Spc110p affects the localization of this protein at the SPB. SPBs from spc110-407 cells were stained with the anti-NSpc110p antibody. The localization of gold particles with sub- structures of Spc110-407p SPBs (Fig. 4E and F) was as described for wild-type SPBs (Fig. 4A-D), indicating that CaM is not required for the proper localization of Spc110p at the SPB. In summary, we conclude that the amino terminus of Spc110p is associated with the inner plaque of the SPB.

\section{CaM-binding to synthetic Spc110p peptides}

To further characterize the interaction of CaM with Spc110p, we studied the binding of purified $\mathrm{CaM}$ to synthetic peptides corresponding to the CaM-binding site of Spc110p by a tryptophan fluorescence assay. The fluorescent amino acid tryptophan was introduced for leucine in positions 3 (peptide 1) or 10 (peptide 2) of the synthetic Spc110p-peptides (Fig. 5A). To avoid artefacts of disulphide bond formation by the cysteine in position 15, this amino acid was replaced in peptide 2 by a serine residue. Peptides 1 and 2 showed a strong fluorescent signal with a maximum at $350 \mathrm{~nm}$ (Fig. 5B) when excited at $295 \mathrm{~nm}$. In contrast, our purified CaM, which does not contain the amino acid tryptophan (Davis et al., 1986), gave no fluorescent signal in this assay (data not shown). Upon binding of $\mathrm{CaM}$ to the Spc110p-peptides, the environment of the trypto- 
phan in the peptides changed, resulting in a blue-shift in the emission spectrum maximum from $350 \mathrm{~nm}$ to $345 \mathrm{~nm}$ (Fig. $5 \mathrm{~B})$. This was accompanied by an increase in fluorescence intensity. The changes of the fluorescence intensity at $330 \mathrm{~nm}$ upon $\mathrm{CaM}$ binding to the peptides were used to calculate the dissociation constant $\left(K_{\mathrm{d}}\right)$ of the binding reaction by Scatchard analysis. Both peptides bound to $\mathrm{CaM}$ with high affinity in the nanomolar range (Fig. 5A). The $K_{\mathrm{d}}$ of the binding reaction was independent of the $\mathrm{Ca}^{2+}$ concentration for peptide 1. A moderate twofold increase in $K_{\mathrm{d}}$ (lower affinity) for peptide 2 was measured when $\mathrm{Ca}^{2+}$ was removed by the $\mathrm{Ca}^{2+}$ chelator EGTA, compared to the affinity in the presence of $39.8 \mu \mathrm{M}$ free $\mathrm{Ca}^{2+}$. For both peptides, the $K_{\mathrm{d}}$ of the binding reaction was unaltered for free $\mathrm{Ca}^{2+}$ concentrations up to $1 \mathrm{mM}$ (data not shown). The stoichiometry of the binding reactions was close to one $(n=0.9 \pm 0.3)$ indicating that one peptide bound to one $\mathrm{CaM}$ molecule. CaM-binding studies using a synthetic substrate (KKRELIESKWHRLLFHDKK) of the related Cdc31p revealed a $K_{\mathrm{d}}$ above $10 \mu \mathrm{M}$ (A.S., unpublished), suggesting that high affinity binding of $\mathrm{CaM}$ is specific for the Spc110p-peptides.

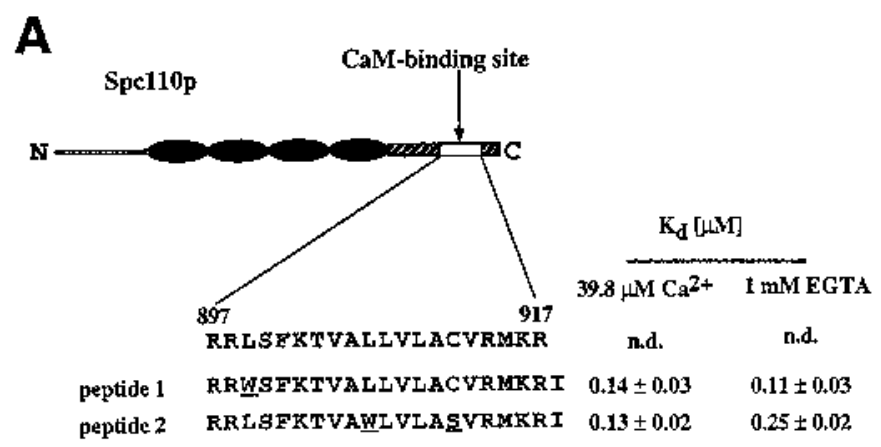

B

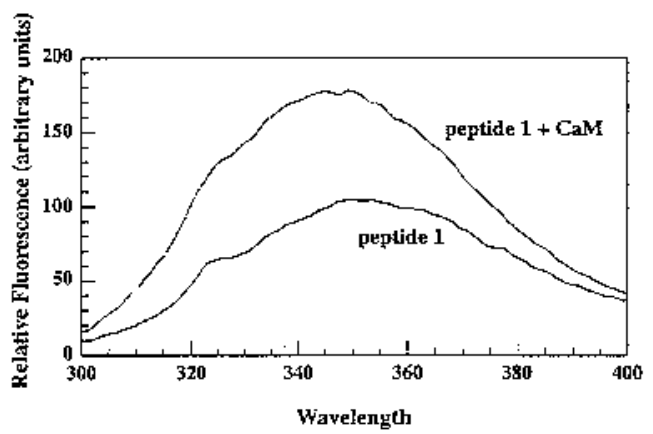

Fig. 5. The binding of CaM to the CaM-binding site of Spc110p. (A). The amino acid sequences of synthetic peptides 1 and 2 relative to Spc110p are shown. Peptide 1 carries a L3W and peptide 2 a L10W and a C15S exchange. Increasing concentrations of $\mathrm{CaM}(0.1$ to $4 \mu \mathrm{M})$ were titrated to peptides 1 and $2(1 \mu \mathrm{M})$. The change in fluorescence intensity at $330 \mathrm{~nm}$ was used to calculated binding curves. The $K_{\mathrm{d}}$ of the binding reaction was determined by Scatchard analysis (Scatchard, 1949). The $K_{\mathrm{d}}$ was measured in the presence of either $39.8 \mu \mathrm{M} \mathrm{Ca}^{2+}$ or $1 \mathrm{mM}$ EGTA. The average of three independent experiments is indicated. (B) Blue shift of the fluorescence emission maximum of peptide $1(1 \mu \mathrm{M})$ upon CaM binding $(4 \mu \mathrm{M})$ from $350 \mathrm{~nm}$ to $345 \mathrm{~nm}$. In addition the relative fluorescence intensity increased. Note, since $\mathrm{CaM}$ does not contain the amino acid tryptophan, it does not contribute to the fluorescence signal.

\section{DISCUSSION}

\section{Spc110p directs CaM via its CaM-binding site to the central plaque of the SPB}

The SPB is a complex structure embedded in the nuclear envelope, with essential functions in chromosome segregation and nuclear migration during mitosis and meiosis, in nuclear fusion during karyogamy and in spore wall formation at the end of meiosis (Byers, 1981; Byers and Goetsch, 1975). Despite these essential functions during the entire life cycle of a yeast cell, only a few SPB components have been identified and localized to substructures (Fig. 6). For example, Cdc31p and Karlp have been localized to the half bridge of the SPB (Spang et al., 1993, 1995), which has important functions in SPB duplication. Other SPB substructures are fibres connecting the central and inner plaques. These fibres consist of at least one SPB protein, Spc110p (Kilmartin et al., 1993).

$\mathrm{CaM}$ is a protein with diverse essential functions in yeast (Ohya and Botstein, 1994). A fraction of CaM is associated with the SPB (Geiser et al., 1993; Stirling et al., 1994). In agreement with this localization, some CMD1 mutants cause defects in SPB functions and nuclear integrity (Ohya and Botstein, 1994). The defects in SPB duplication may be the consequence of a reduced interaction of the mutated $\mathrm{CaM}$ with Spc110p, which contains a CaM-binding site at its carboxyterminal end (Geiser et al., 1993; Stirling et al., 1994). Therefore it was important to determine whether CaM is directed to the SPB via Spc110p. Our results suggest that Spc110p is the major and probably the only CaM-binding

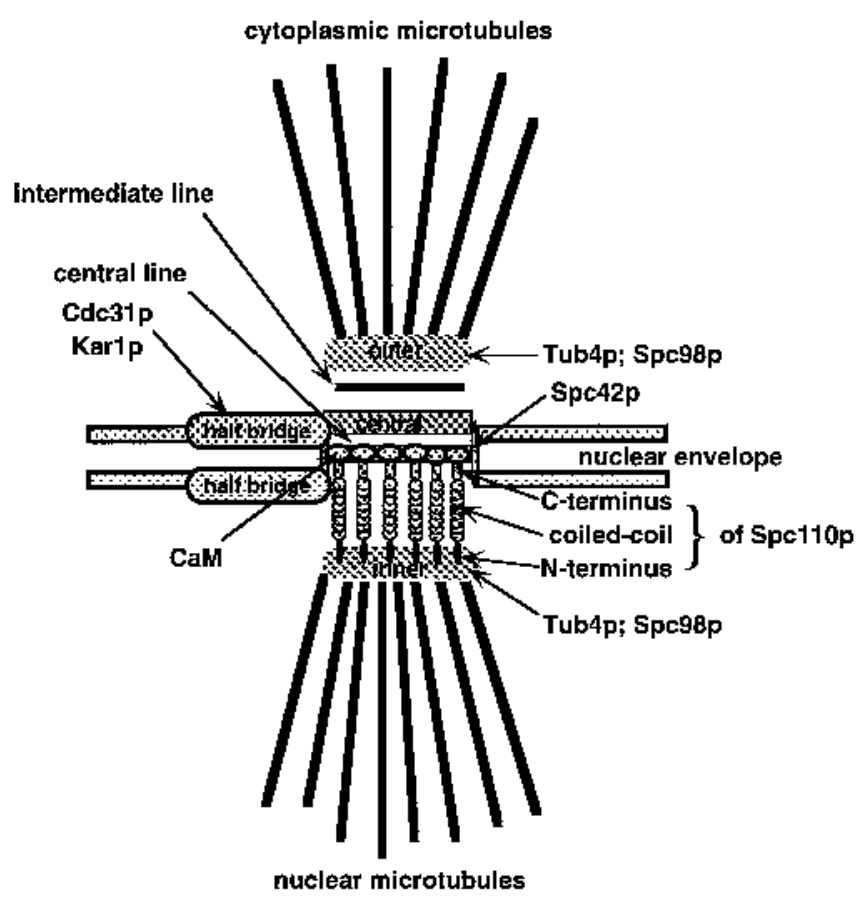

Fig. 6. Schematic diagram showing the localization of $\mathrm{CaM}$ and indicating the direction of Spc110p at the SPB, relative to other known SPB components. The localization of Cdc31p (Spang et al., 1993), Kar1p (Spang et al., 1995), Spc98p (Rout and Kilmartin, 1990; Geissler et al., 1996), Tub4p (Spang et al., 1996), Spc42p (Donaldson and Kilmartin, 1996), CaM and Spc110p (Kilmartin et al., 1993) are shown. 
protein at the SPB. This conclusion is based on the finding that the association of CaM with the central plaque of the SPB is dependent on the CaM-binding site of Spc110p (Fig. 6). However, we were unable to show an interaction of CaM with Spc110p by immunoprecipitation using anti-Spc110p antibodies or a functional protein A-Spc110p hybrid protein (data not shown). This may be explained by the dissociation of $\mathrm{CaM}$ from Spc110p during the precipitation procedure.

The function of CaM at the SPB is not understood, especially since deletion of the CaM binding site of Spc110p does not cause an obvious growth defect (Geiser et al., 1993) or a defect in SPB structure (this study). In contrast, point mutations in the CaM-binding site of Spc110p that reduced CaM-binding in vitro affected viability of cells (Stirling et al., 1994). Therefore, it was suggested that the carboxy-terminal domain of Spc110p has an inhibitory function, which is either relieved by deletion of this domain or by the binding of CaM (Stirling et al., 1994). Interestingly, while the entire Spc110p does not interact with $\mathrm{CaM}$ in the two-hybrid system the carboxy-terminal domain of Spc110p does (Geiser et al., 1993; E.S., unpublished). This may indicate that the CaM-binding site of Spc110p is buried in the nuclear form of Spc110p but becomes accessible after binding to other proteins. One possibility is that CaM ensures the correct assembly of the SPB after Start of the cell cycle (Byers, 1981), by binding to Spc110p.

\section{Spc110p spans the inner and central plaques with the carboxy terminus embedded in the central plaque}

It has been proposed that Spc110p functions as a spacer protein spanning the inner and central plaques of the SPB. This model is based on the finding that deletions in the coiled-coil domain of Spc110p corresponded to changes in distance between the inner and central plaques of the SPB (Kilmartin et al., 1993). A logical consequence of such a model is the association of one end of Spc110p with the central plaque and the other with the inner plaque of the SPB. This is consistent with our results, indicating that the association of $\mathrm{CaM}$ with the central plaque is dependent on the CaM-binding site of Spc110p. Since the latter is located near the carboxy terminus of Spc110p, we suggest that this part of Spc110p is close to the central plaque (Fig. 6). Confirming the extended nature of Spc110p, antibodies directed against the amino-terminal 175 amino acids of Spc110p labelled the inner plaque of the SPB. Taken together, our results confirm that Spc110p is an extended molecule at the SPB and in addition, they define the topology of Spc110p with the amino terminus directed towards the inner plaque and the carboxy terminus associated with the central plaque of the SPB (Fig. 6).

In agreement with other reports, we found a fraction of Spc110p within the nucleus of yeast cells (Kilmartin et al., 1993). Since our affinity-purified anti-Spc110p antibodies are highly specific for Spc110p (Spang et al., 1995) and the same signal was obtained by rabbit and goat anti-N-Spc110p antibodies, we are confident that we detected Spc110p and not a cross-reactive protein. The nuclear Spc110p may be a precursor of the SPB associated form. Alternatively, Spc110p could have additional functions e.g. in organizing the nucleus.

\section{CaM binds to Spc110p with high affinity but independent of $\mathrm{Ca}^{2+}$}

We measured the binding of CaM to synthetic peptides corre- sponding to the CaM-binding site of Spc110p. The CaM binding region of Spc110p has been defined by a deletion analysis (Geiser et al., 1993) as well as by mutations in Spc110p affecting the in vitro binding of CaM (Stirling et al., 1994). The minimal region of Spc110p which is required for the interaction with CaM seems to span amino acids 901-914 (Stirling et al., 1994). The peptides used in our binding study corresponded to amino acids 897-917 of Spc110p and therefore included the entire CaM-binding site of Spc110p. For our measurements the amino acid leucine of the Spc110p-peptide was changed to tryptophan to allow monitoring of the binding of $\mathrm{CaM}$ to the peptides by changes in fluorescence. Similar modifications have been introduced into the CaM-binding sites of myosin light chain kinase and affect the affinity of the binding reaction mostly by factors of 0.7 to 2 (O'Neil et al., 1987). This, and the fact that we obtained very similar $K_{\mathrm{d}}$ values for both Spc110p-peptides, suggest that our results reflect the behaviour of the unmodified peptide.

To our knowledge, we report the first quantitative binding studies for yeast calmodulin. The stoichiometry of the reaction was close to one, indicating that one calmodulin bound to one Spc110-peptide. The $K_{\mathrm{d}}$ of the binding reaction was in the 100 $\mathrm{nM}$ range for both peptides. This is relatively high compared to the $1 \mathrm{nM}$ to $10 \mathrm{nM} K_{\mathrm{d}}$ values determined for the binding of target peptides to vertebrate calmodulin (O'Neil et al., 1987). We do not think that the peptide length of 21 amino acids is responsible for the lower affinity binding since this is above what has been used in comparable studies with vertebrate calmodulin (17 to 20 amino acids; O'Neil et al., 1987). Whether the lower affinity binding of $\mathrm{CaM}$ to target proteins is a general feature in yeast or a specific property of the CaMSpc110p interaction remains to be determined. Since $\mathrm{Ca}^{2+}$ is not an essential modulator for CaM's binding activity in yeast (Geiser et al., 1991), lower affinity binding of CaM may be required to ensure the dissociation of $\mathrm{CaM}$ from target proteins.

We found that the $K_{\mathrm{d}}$ of CaM-peptide binding was not influenced (peptide 1) or only slightly influenced (twofold; peptide 2) by $\mathrm{Ca}^{2+}$. $\mathrm{Ca}^{2+}$-independent binding of $\mathrm{CaM}$ was expected on the one hand, since high-affinity $\mathrm{Ca}^{2+}$ binding is evidently not required for calmodulin's essential functions (Geiser et al., 1991). On the other hand the interaction of CaM with filterimmobilized Spc110p was dependent on $\mathrm{Ca}^{2+}$ (Spang et al., 1995). These contrary results may be explained by the denatured state of Spc110p in the filter binding assay. Alternatively, amino acids flanking the CaM-binding site of Spc110p, which are not present in peptides 1 and 2, may mediate $\mathrm{Ca}^{2+}$-dependent interaction with $\mathrm{CaM}$ in vivo.

We thank Dr Euteneuer-Schliwa for the WA3 antibody and Dr Davis for plasmid pJG94. J. Murphy is gratefully acknowledged for helping with the confocal laser microscope.

\section{REFERENCES}

Adams, A. E. M. and Pringle, J. (1989). Relationship of actin and tubulin to budgrowth in wild-type and morphogenetic-mutant Saccharomyces cerevisiae. J. Cell Biol. 108, 934-945.

Ausubel, F. M., Brent, R., Kingston, R. E., Moore, D. D., Seidman, J. G., Smith, J. A. and Struhl, K. (1994). Current Protocols in Molecular Biology. John Wiley and Sons, New York. 
Brockerhoff, S. E., and Davis, T. N. (1992). Calmodulin concentrates at regions of cell growth in Saccharomyces cerevisiae. J. Cell Biol. 118, 619629.

Bradford, M. M. (1976). A rapid and sensitive method for the quantitation of microgram quantities of protein utilizing the principle of protein-dye binding. Anal. Biochem. 72, 248-254.

Byers, B. (1981). Cytology of the Yeast Life Cycle. Cold Spring Harbor Laboratory Press, Cold Spring Harbor, NY.

Byers, B. and Goetsch, L. (1975). Behavior of spindles and spindle plaques in the cell cycle and conjugation of Saccharomyces cerevisiae. J. Bacteriol. 124, 511-523.

Cohen, P. and Klee, C. B. (1988). Calmodulin. Elsevier Science Publishers BV, Amsterdam, The Netherlands.

Davis, T. N. (1992). Mutational analysis of calmodulin in Saccharomyces cerevisiae. Cell Calcium 13, 435-444.

Davis, T. N., Urdea, M. S., Masiarz, F. R. and Thorner, J. (1986). Isolation of the yeast calmodulin gene: Calmodulin is an essential protein. Cell 47, 423-431.

Donaldson, A. D. and Kilmartin, J. V. (1996). Spc42p: Phosphorylated component of the S. cerevisiae spindle pole body (SPB) with an essential function during SPB duplication. J. Cell Biol. 132, 887-901.

Geiser, J. R., Sundberg, H. A., Chang, B. H., Muller, E. G. D. and Davis, T. N. (1993). The essential mitotic target of calmodulin is the 110-kilodalton component of the spindle pole body in Saccharomyces cerevisiae. Mol. Cell. Biol. 13, 7913-7924.

Geiser, J. R., Tuinen, V. D., Brockerhoff, S. E., Neff, M. M. and Davis, T. N. (1991). Can calmodulin function without binding calcium? Cell 65, 949-959.

Geissler, S., Pereira, G., Spang, A., Knop, M., Souès, S., Kilmartin, J. and Schiebel, E. (1996). The spindle pole body component Spc98p interacts with the $\gamma$-tubulin-like Tub4p of $S$. cerevisiae at the sites of microtubule attachment. EMBO J. (in press).

Guthrie, C. and Fink, G. R. (1991). Guide to Yeast Genetics and Molecular Biology. Academic Press, New York.

Harlow, E. and Lane, D. (1988). Antibodies. Cold Spring Harbor Laboratory Press, Cold Spring Harbor, NY.

Kilmartin, J. V., Dyos, S. L., Kershaw, D. and Finch, J. T. (1993). A spacer protein in the Saccharomyces cerevisiae spindle pole body whose transcription is cell-cycle regulated. J. Cell Biol. 123, 1175-1184.

Laemmli, U. K. (1970). Cleavage of structural proteins during the assembly of the head of bacteriophage T4. Nature 227, 680-685.

Mirzayan, C., Copeland, C. S. and Snyder, M. (1992). The NUF1 gene encodes an essential coiled-coil related protein that is a potential component of the yeast nucleoskeleton. J. Cell Biol. 116, 1319-1332.

Ohya, Y. and Botstein, D. (1994). Diverse essential functions revealed by complementing yeast calmodulin mutants. Science 263, 963-966.
O'Neil, K. T., Wolfe, H. R., Erickson-Viitanen, J. S. and Degrado, W. F. (1987). Fluorescence properties of calmodulin-binding peptides reflect alpha-helical periodicity. Science 236, 1454-1456.

Preuss, D., Mulholland, J., Kaiser, C. A., Orlean, P., Albright, C., Rose, M. D., Robbins, P. W. and Botstein, D. (1991). Structure of the yeast endoplasmic reticulum: Localization of ER proteins using immunofluorescence and immunoelectron microscopy. Yeast 7, 891-911.

Rothstein, R. J. (1983). One-step gene disruption in yeast. Meth. Enzymol. 101, 202-211.

Rout, M. P. and Kilmartin, J. V. (1990). Components of the yeast spindle and spindle pole body. J. Cell Biol. 111, 1913-1927.

Sambrook, J., Fritsch, E. F. and Maniatis, T. (1989). Molecular Cloning. Cold Spring Harbor Laboratory, Cold Spring Harbor.

Scatchard, G. (1949). The attractions of proteins for small molecules and ions. Ann. NY Acad. Sci. 51, 660-672.

Schulman, H., Hanson, P. I. and Meyer, T. (1992). Decoding calcium signals by multifunctional CAM kinase. Cell Calcium 13, 401-411.

Sikorski, R. S. and Hieter, P. (1989). A system of shuttle vectors and yeast host strains designed for efficient manipulation of DNA in Saccharomyces cerevisiae. Genetics 122, 19-27.

Spang, A., Courtney, I., Fackler, U., Matzner, M. and Schiebel, E. (1993). The calcium-binding protein cell division cycle 31 of Saccharomyces cerevisiae is a component of the half bridge of the spindle pole body. J. Cell Biol. 123, 405-416.

Spang, A., Courtney, I., Grein, K., Matzner, M. and Schiebel, E. (1995). The Cdc31p-binding protein Karlp is a component of the half-bridge of the yeast spindle pole body. J. Cell Biol. 128, 863-877.

Spang, A., Geissler, S., Grein, K. and Schiebel, E. (1996). The $\gamma$-tubulin-like Tub4p of Saccharomyces cerevisiae is associated with the spindle pole body substructures that organize microtubules and is required for mitotic spindle formation. J. Cell Biol., in press.

Stirling, D. A., Welch, K. A. and Stark, M. J. R. (1994). Interaction with calmodulin is required for the function of Spc110p, an essential component of the yeast spindle pole body. EMBO 13, 4329-4342.

Studier, F. W. and Moffat, B. A. (1986). Use of bacteriophage T7 RNA polymerase to direct selective high level expression of cloned genes. J. Mol. Biol. 189, 113-130.

Tabor, S. and Richardson, C. C. (1985). A bacteriophage T7 RNA polymerase/promotor system for controlled exclusive expression of specific genes. Proc. Natl. Acad. Sci. 82, 1074-1078.

Yano, K., Araki, Y., Hales, S. J., Tanaka, M. and Ikebe, M. (1993). Boundary of the autoinhibitory region of smooth-muscle myosin light-chain kinase. Biochemistry 32, 12054-12061.

(Received 8 March 1996 - Accepted 31 May 1996) 